\section{Author Reply: Surgery in Stage IV Breast Cancer Patients: Continued Controversy}

We appreciate the opportunity to respond to Dr. Fitzal's letter to this journal regarding our 2008 study evaluating the impact of timing on surgical intervention at the primary tumor site in Stage IV breast cancer. ${ }^{1}$ The limitations of any retrospective analysis are well known; currently, however, these are the data available to guide treatment decisions and future trials. In this light, there are multiple retrospective single-institution and database reviews that indicate a possible survival advantage. ${ }^{2-5}$ Included in these are both our initial analysis comparing surgical intervention and systemic therapy versus systemic therapy alone, ${ }^{2}$ as well as the Fields' et al. publication supporting our initial findings. ${ }^{4}$

To our knowledge, the surgical intervention timing study is the only one to carefully evaluate a cohort of patients treated at a single institution and identify factors that contributed to increased survival among the patients who received surgical intervention. ${ }^{1}$ Timing of surgical intervention did not contribute to survival on multivariate analysis, a point discussed extensively; however, there was an improvement in metastatic progression-free survival $(P=.0008)$ on univarate analysis. Estrogen receptor and progesterone receptor status were evaluated and not found to be contributing factors to survival $(P=.74, .339$, respectively) or metastatic progression-free survival $(P=.796, .952$ respectively $)$ and therefore not reported. The study from Fields et al. does evaluate why surgical intervention was performed-diagnosis, curative intent, or palliation; however, there is no specific evaluation of the timing of surgical intervention from the date of diagnosis and its correlation with survival.

In reference to the ongoing study of Dr. Badwe, the group at Tata Memorial is congratulated for being able to initiate a trial that has been discussed in the United States for years, but unfortunately not performed. Their preliminary analysis of 99 patients suggests an improvement in progression-free survival in patients with local resection. ${ }^{6}$ All patients were randomized only AFTER completing chemotherapy with cyclophosphamide, 5FU, and epirubicin; therefore, most likely placing them in the same group that had improved metastatic progression-free survival in our review-those patients who had surgery $>3$ months after their initial diagnosis of breast cancer. Patients in this initial dataset were not randomized prior to initiation of systemic therapy.

Improving systemic therapies have enabled increased survival in Stage IV patients, underlining the need for prospective data to optimize patient outcomes. ${ }^{7} \mathrm{We}$ look forward to the results of Dr. Badwe's trial, which plans to have an enrollment of 350 patients and should be adequately powered to answer this important question.

\section{Roshni Rao, MD ${ }^{1}$, and Gildy Babiera, MD $^{2}$}

${ }^{1}$ Division of Surgical Oncology, Department of Surgery, University of Texas Southwestern Medical Center, Dallas, TX;

${ }^{2}$ Surgical Oncology, UTMD Anderson Cancer Center, Houston, TX

e-mail: Roshni.Rao@utsouthwestern.edu

Published Online: 25 February 2009

(C) Society of Surgical Oncology 2009

\section{REFERENCES}

1. Rao R, Feng L, Kuerer HM, Singletary SE, Bedrosian I, Hunt KK, et al. Timing of surgical intervention for the intact primary in stage IV breast cancer patients. Ann Surg Oncol. 2008;15:1696-702.

2. Khan SA, Stewart AK, Morrow M. Does aggressive local therapy improve survival in metastatic breast cancer? Surgery. 2002;132: $620-7$.

3. Babiera GV, Rao R, Feng L, Meric-Bernstam F, Kuerer HM, Singletary SE, et al. Effect of primary tumor extirpation in breast cancer patients who present with stage IV disease and an intact primary tumor. Ann Surg Oncol. 2006; 13:776-82.

4. Fields RC, Jaffe DB, Trinkaus K, Zhang Q, Arthur C, Aft R, et al. Surgical resection of the primary tumor is associated with increased long-term survival in patients with stage IV breast cancer after controlling for site of metastasis. Ann Surg Oncol. 2007; $14: 3345-51$.

5. Blanchard DK, Shetty PB, Hilsenbeck SF. Association of surgery with improved survival in stage IV breast cancer patients. Ann Surg. 2008;247:732-8.

6. Badwe RA, Hawaldar RW, Khare A, Nadkarni M, Jalali R, Parmar $\mathrm{V}$. Role of local-regional treatment in metastatic breast cancer at presentation: A randomized trial. San Francisco, CA: In ASCO Breast Cancer Symposium; 2008.

7. Andre F, Slimane K, Bachelot T, Dunant A, Namer M, Barrelier A, et al. Breast cancer with synchronous metastases: trends in survival during a 14 year period. J Clin Oncol. 2004;22:3302-8. 